\title{
Incidental findings of a bilateral complete ossification of stylo-hyoid chain and thyro-hyoid ligaments.
}

eya moussaoui ${ }^{1}$, Imen Chaabani ${ }^{1}$, Kadri Sahar ${ }^{2}$, lamia oualha ${ }^{1}$, and Nabiha Douki ${ }^{1}$

${ }^{1}$ University of Monastir Faculty of Dental Medicine of Monastir

${ }^{2}$ University of Monastir

December 5, 2021

\author{
Abstract \\ Ossification of stylohyoid chain corresponds to the ossification of stylohyoid ligament that can vary from thin short to thick long \\ ossification and can be associated with other calcifications. We report in this case a bilateral non painful complete ossification \\ of the stylohyoid complex extended to the thyrohyoidien ligament. \\ Title: Incidental finding of a bilateral complete ossification of stylo-hyoid chain and thyro-hyoid \\ ligaments. \\ Moussaoui eya [1,2], imen chaabani [3], Sahar kadri [1,2], Oualha Lamia [1], Douki Nabiha [1,2] \\ 1: Department of dental medicine, SAHLOUL Hospital (Sousse), Dental Faculty of Monastir, University of \\ Monastir, Tunisia. \\ 2: Laboratory of oral health and maxillofacial rehabilitation (LR12ES11), University of Monastir, Tunisia. \\ 3: Department of radiology, university dental clinic,University of Monastir, Tunisia
}

\section{Corresponding author:}

Eya MOUSSAOUI : mail address: eyamouss@gmail.com Address: SAHLOUL Hospital, Department of dental medicine, Sousse, Tunisia.

Assistant professor at the department of dental medicine, Department of dental medecine, SAHLOUL Hospital (Sousse), University of Monastir.

Co- authors:

Imen chaabani: mail address: chaabanimen@gmail.com

Aggregate professor at the department of radiology, university dental clinic,University of Monastir, Tunisia

Sahar kadri: mail address: kadrisahar1995@gmail.com

Resident at the department of dental medicine, Department of dental medecine, SAHLOUL Hospital (Sousse), University of Monastir.

*Lamia OUALHA : mail address: lamia.oualha@gmail.com Address: SAHLOUL Hospital, Department of dental medecine, Sousse, Tunisia.

Professor at the department of dental medicine, SAHLOUL Hospital (Sousse), University of Monastir. 
*Nabiha DOUKI : mail address: nabiha.douki@gmail.com Address: SAHLOUL Hospital, Department of dental medecine, Sousse, Tunisia.

Professor and head of department of dental medicine, SAHLOUL Hospital (Sousse), University of Monastir.

Patient consent:

I confirm that written patient consent has been signed and collected in accordance with the journal's patient consent policy.

\section{Author contribution:}

Eya moussaoui: data collection and interpretation and drafting the manuscript

Imen Chaabani: aquisation and analysis of data and drafting the manuscript

Sahar kadri: collection and interpretation of data

Ouaha lamia: revising the manuscript for important intellectual content

Nabiha douki: conception and design and revising the manuscript

Abstract:

Ossification of stylohyoid chain corresponds to the ossification of stylohyoid ligament that can vary from thin short to thick long ossification and can be associated with other calcifications. We report in this case a bilateral non painful complete ossification of the stylohyoid complex extended to the thyrohyoidien ligament.

Key clinical message: Ossification and elongation of the stylohyoid chain can cause neck and facial symptoms, known as Eagle's syndrome. Nonetheless, a bilateral ossified and elongated stylohyoid chain associated with ossification of the thyro-hyoid ligament may be not painful.

Keywords: stylohyoid ligament, eagle syndrome, tyro-hyoid ligament, styloid chain, pseudarthroses

A 53-year-old man consulted the dental medicine department for a dental check up. A routine conventional dental radiographic exam was performed and showed bilateral ossification of the stylohyoid complex (figure 1), then confirmed by a lateral neck x-ray (figure 2) that showed ossification of the styloid chain (Styloid Process, stylohyoid ligament and lesser cornua of the hyoid bone) extended to the thyrohyoidien ligament .

The patient had no pain, with very restricted head and neck movements.

He reported the history of severe cervical trauma caused by a fall down stairs 30 years ago.

CT scans of the neck showed an elongated and heavily ossified styloid process, the stylohyoidien ligament extending to the lesser cornua of the hyoid bone with three pseudarthroses, associated with a bilateral ossification of the thyrohyoid ligaments (figure 3).

Usually, the mineralization of the stylohoid ligament complex cause oro-facial pain defining the Eagle's syndrome $[1,2]$, however it can be non painful and discovered incidentally as in our case. A previous cervical trauma or surgery has been frequently reported as a the main cause [2].

[1] Chebbi R et al. Elongated styloid process as a cause of facial pain joint bone spine. 2014 Jul;81(4):368. doi: 10.1016/j.jbspin.2014.03.007.

[2] Y. Sudrat et al. Eagle's syndrome: a case report with multiples calcifications. Med Buccale Chir Buccale 2008; $14:$ 97-102. 

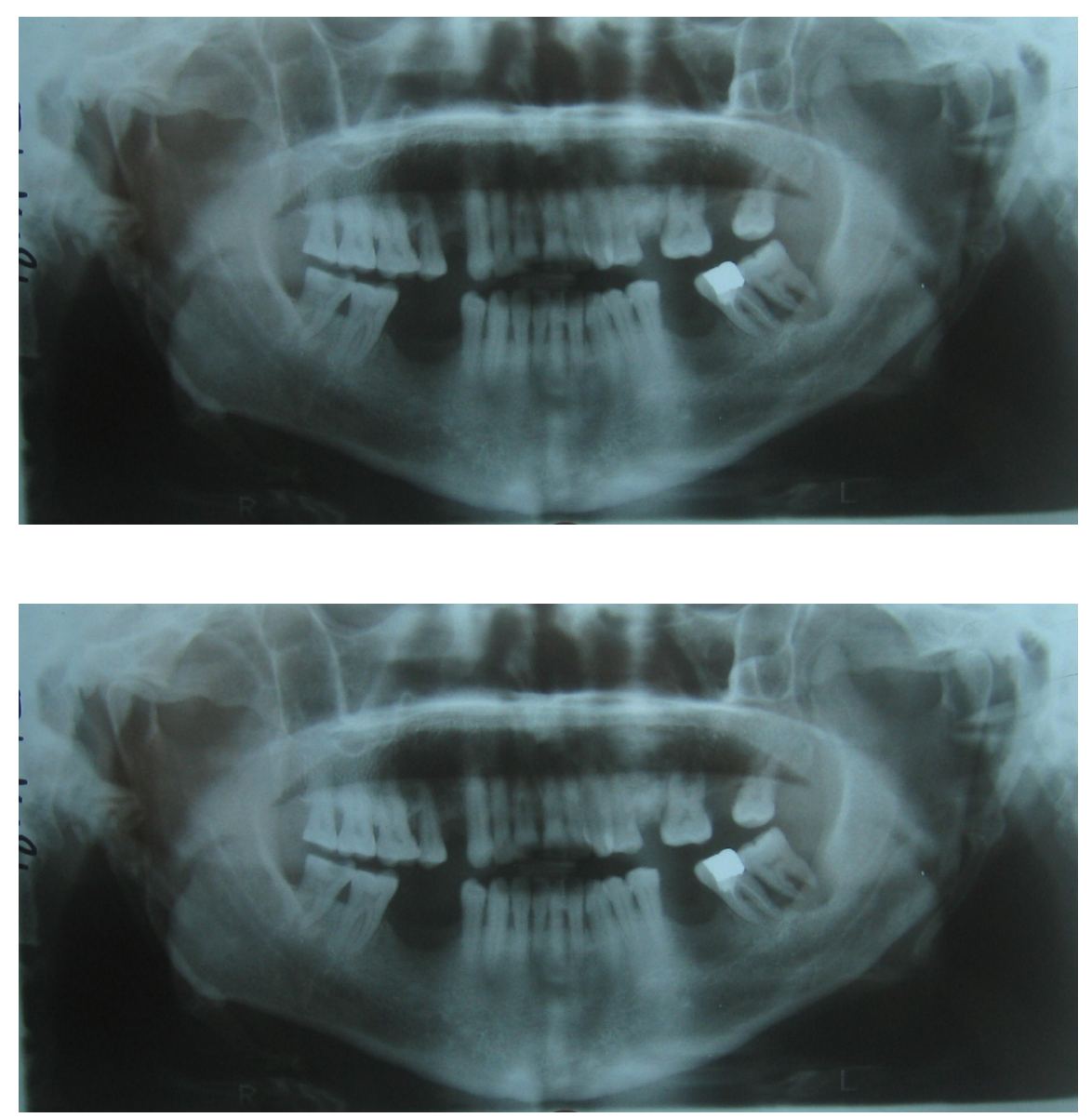

Fig 1: bilateral ossification of the stylohyoid complex visualized in the panoramic xray

Fig CT scan (3D VRT) of the neck shows complete ossified styloid chain and thyrohyoid ligament . Styloid process

Hyoid bone

Ossification of thyrohyoid ligament

Pseudoarthroses 


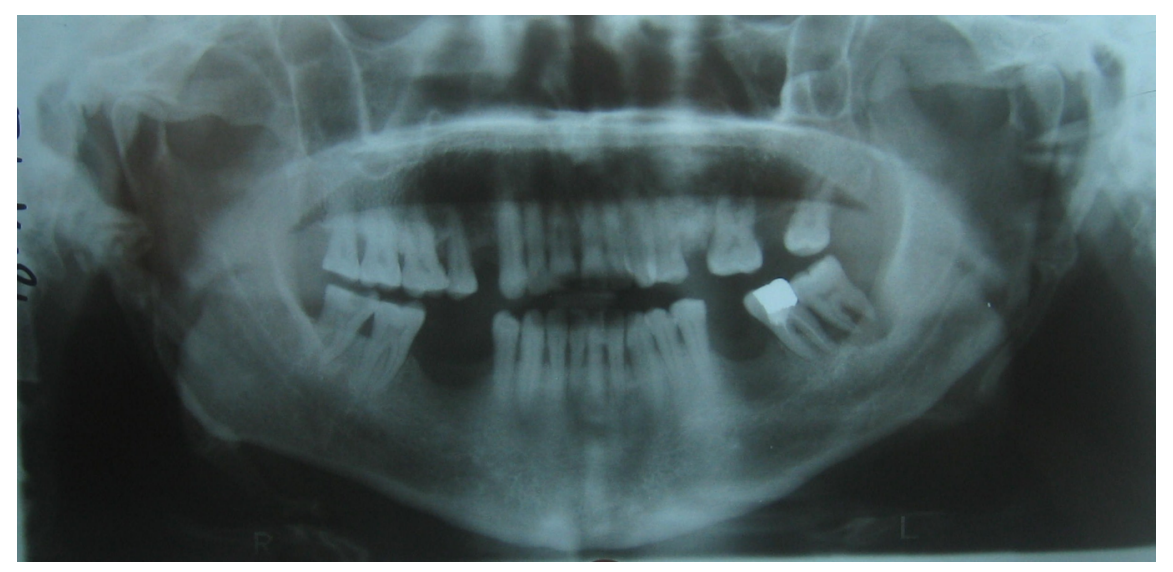

\section{Conflicts of interest:}

All the authors declare not having any conflicts of interest.

\section{Ethics statement:}

Data from the patient included in this case report were treated anonymously and a statement of informed consent was signed to allow the use of her medical and dental records and photos.

\section{Funding:}

The authors received no financial support for the research, authorship, and/or publication of this article.

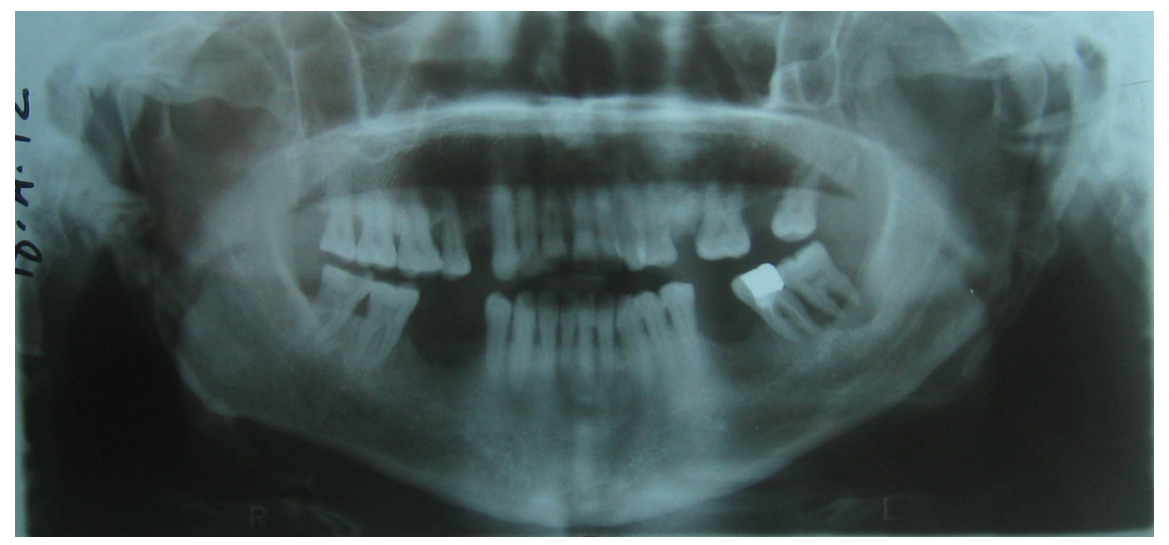



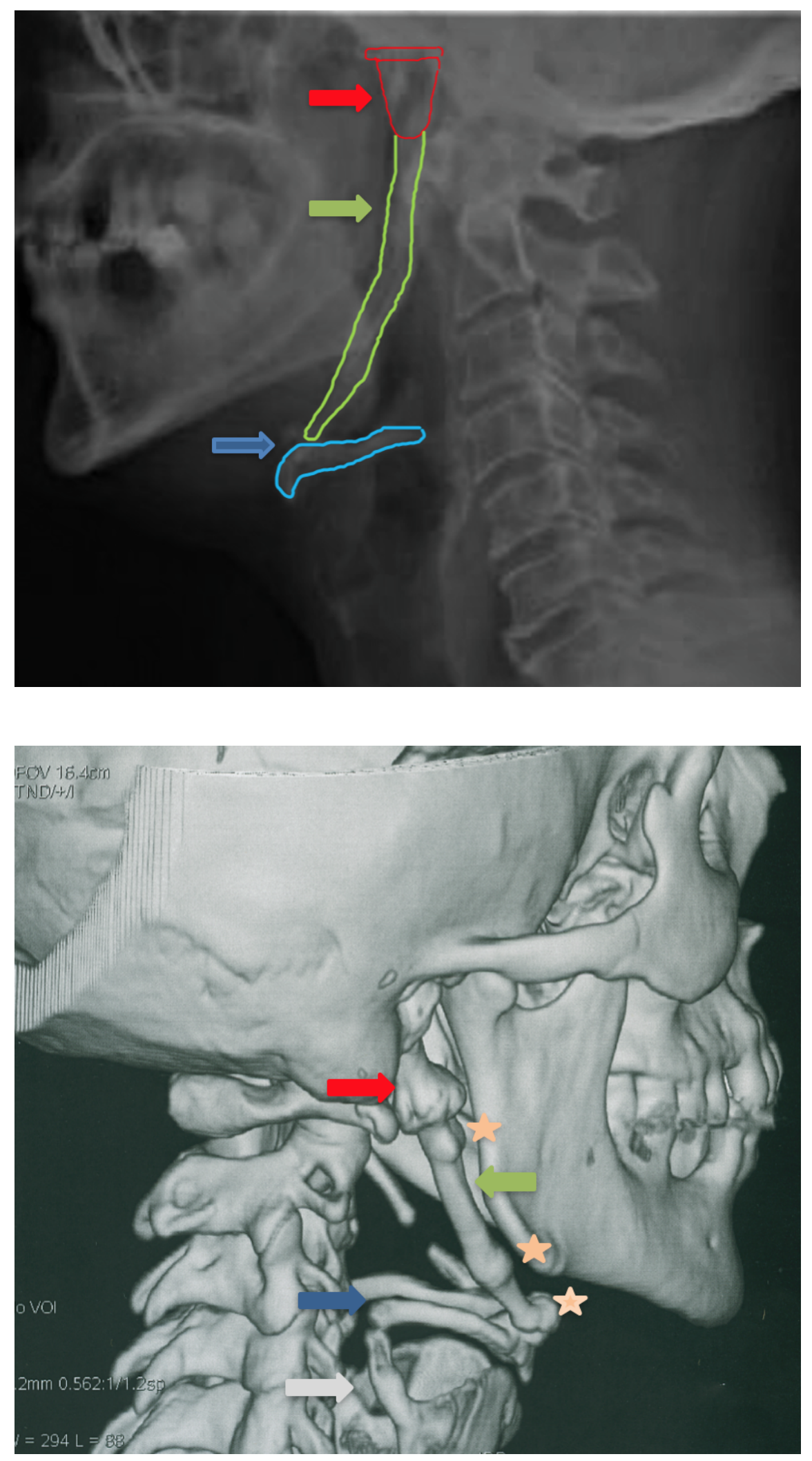